\title{
Study on Projectile Shape's Influence in Rotary Penetration Depth of Concrete Target
}

\author{
J. Guo,.C. Pan,Y. He,C.X. Pang,.J. Deng \\ Ministerial Key laboratory of ZNDY, Nanjing University of Science \& Technology, Nanjing \\ 210094,China
}

\begin{abstract}
KEYWORD: Concrete; Rotary Penetration; Scored projectile; Numerical simulation ABSTRACT: To study the effect of the projectile shape's influence in rotary penetration depth(RDP) of concrete target, LS-DYNA was used to simulate the process of the projectile rotary penetrating in the target under different projectile shape and different velocities. Simulation results prove that drilling-head shape projectile could increase RDP with low velocity, when ogive-nosed shape projectile could do well with high velocity, so composed of drilling-head and ogive-nosed shape could improve penetration performance; when rotary velocity is $50000 \mathrm{r} / \mathrm{min}$, the composed shape projectile the most optimal increasing rate of RDP with the axis velocity of $300-400 \mathrm{~m} / \mathrm{s}$. Under excellent loading condition, the depth of the cutting slot has the larger effect on penetration depth than the slope, the cutting slot shape that relatives to target surface has little effect of the penetration. Based on the validation test of penetrating into targets, the simulation results are coordination with the experiment data.
\end{abstract}

\section{INTRODUCTION}

As the targets of military importance become more and more solid and underground, kinetic energy penetration weapon has attracted more and more attentions from various countries in the world, and been developed rapidly. To improve the penetration performance of kinetic energy penetration weapon is the main objective of weapon researchers. There are many factors that affect the penetration performance, such as projectile material, head shape, projectile structure, striking velocity, etc. However, rotation, which is an important concomitant process during the penetration process, is usually ignored.

As the concept of digger weapons system been proposed, people gradually began to study the rotation effect during the penetration process. Li[1] carried out the numerical simulation research on the penetration motion of high-speed rotation warhead into the metal sheet. Zhao[2] researched the rotation effect during long rod projectile penetrating semi-infinite thick soil. Pan[3]carried out the experiment and simulation research on the penetration of rotation boosted earth-penetrating projectile into concrete target and the static rotation penetration into concrete target. Pang[4] researched the effect process of rotation grooved projectile into the aluminum target and concrete target.

In order to determine the relationship between the penetration projectile shape and the concrete penetration depth, in this paper, finite element simulation software is used to research the relationship between them via changing the projectile head shape and the penetration speed. The scientificity of the researched is verified via the experiments, and the condition of optimum penetration performance is obtained on this base, which will provide the basis for the design of driving warhead.

\section{NUMERICAL SIMULATION OF THE DRIVING PENETRATION INTO CONCRETE TARGET}

\subsection{Establishment of finite element model}

The finite element program LS-DYNA is used to carry out the numerical simulation, and the model of driving projectile head and target plate are established respectively. For the driving projectile head, free meshing is operated. In order to guarantee the precision of analysis, 8-point 3DSOLID 64 
cell is used to mesh the 3D entity model. The finite element model of the rotation solid is as shown in the Figure 1(a). And the finite element model of the target plate is as shown in the Figure 1(b).

Comprehensively considering the accuracy and time consuming of the calculation, dense grid is used within the center region with a radius of $20 \mathrm{~mm}$, and sparse grid is used in other region. In order to eliminate the influence of reflected stress wave from the back of target plate, non-reflecting boundary condition is set at the back of target plate. Full constraint is set at the side of target plate. The initial rotation is determined by the OMEGA, NX, NY, NZ parameters of the keyword of INITIAL_VELOCITY_GENERATION, among which, OMEGA is used to set the initial angular velocity around a fixed axis, NX, NY and NZ are used to set the rotation around $\mathrm{X}, \mathrm{Y}$ and $\mathrm{Z}$ axis, respectively.

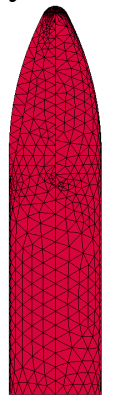

(a) Driving projectile head

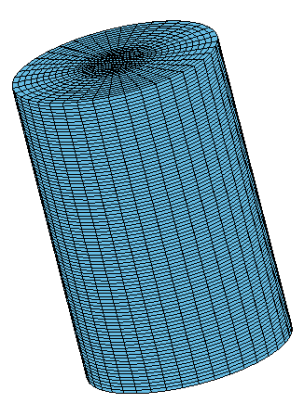

(b) Target plate

Figure 1. Finite element model

\subsection{Related parameters setting}

Holmquist-Johnson-Cook model [5, 6] is selected to be the concrete strength model, which is a dynamic material model developed for the impact loading effect of concrete. The model considers the situation of large strain, high strain rate and high pressure, and combined with the damage theory, considers the tensile fracture behavior of the material, as well as the functional relation between volume compression and pressure after crashing of material. The main related parameters of concrete are set as literature. During the simulation, the driving penetration body is set to be rigid body, and there is no change of the component unit and material during penetration process.

\section{EXPERIMENTAL RESEARCH ON DRIVING PENETRATION INTO CONCRETE}

In order to research the deductive process of driving projectile head shape and the concrete penetration depth, low speed driving projectile head and high speed driving projectile head is designed respectively. Low speed is set to be $0-100 \mathrm{~m} / \mathrm{s}$, and high speed is set to be $100-1000 \mathrm{~m} / \mathrm{s}$. Low speed drilling machine and high speed $14.5 \mathrm{~mm}$ rifle are used to load respectively. The deductive relationship between the penetration projectile shape and the concrete penetration depth is analyzed via changing the experiment condition.

\subsection{Design of driving projectile head}

Two structures are applied for the low speed driving projectile head. One uses drill as an equivalent, the diameter of drill is $15 \mathrm{~mm}$, and the material is high speed steel. The other applies common oval projectile structure. Their structure is shown as in the Figure 2. 

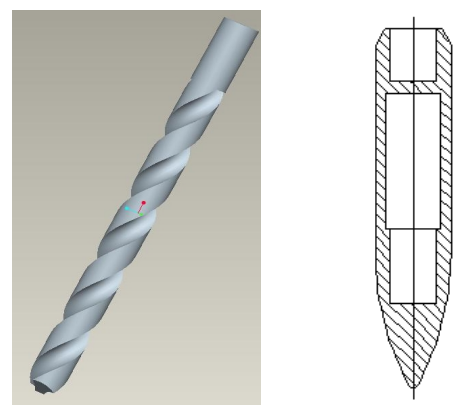

Figure 2. Low speed driving penetration projectile

\subsection{Concrete target}

In this paper, limestone aggregate is selected to be the aggregate of the concrete target in the experiment. The average particle size is $5 \mathrm{~mm}$, PO42.5 grade ordinary portland cement is used, the fine aggregate is made up of coarse quartz stone, cement, sand, coarse aggregate and water with the mixture ratio of 1: 1.2: 2.2: 0.4 . The size of target is $\Phi 300 \mathrm{~mm} \times 200 \mathrm{~mm}$. $3 \mathrm{~mm}$ steel plate is used to band the target. The ratio between the diameter of target and projectile is larger than 20 , so the influence of the boundary of target can be ignored. The target has been cured for 28 days. 3 compression test blocks are processed when pouring two kinds of concrete targets, the dimension is $150 \mathrm{~mm} \times 150 \mathrm{~mm} \times 150 \mathrm{~mm}$. The pouring and curing of the concrete are in accordance with the standard, and the average compressive strength is $46 \mathrm{MPa}$.

\subsection{Layout of experiment}

The drill machine is used to load for the low speed driving penetration, the experiment layout is as shown in Figure 3.

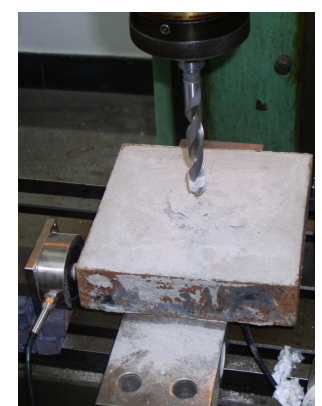

Figure 3. Diagram of low speed driving experiment device

$14.5 \mathrm{~mm}$ rifle is used as the acceleration platform for the high speed driving penetration. The layout of the experiment is as shown in the Figure 4. 5/7 gunpowder is applied to be propellant. The impact velocity of projectile is controlled by adjusting the volume of propellant, and the dual channel tester is used to measure the impact velocity of projectile.

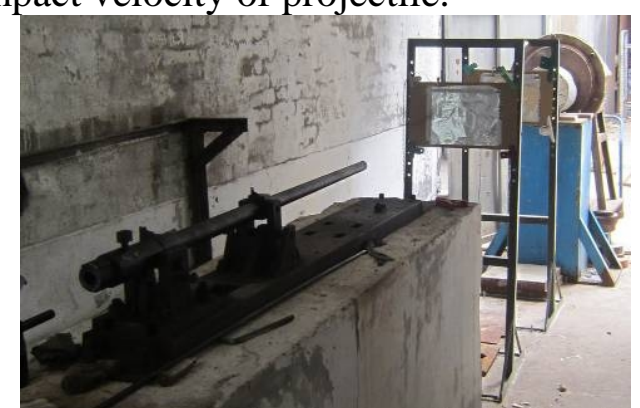

Figure 4. Layout of the dynamic experiment 


\section{EXPERIMENT RESULTS AND ANALYSIS}

\section{DEDUCTIVE PROCESS ANALYSIS OF DRIVING PENETRATION PROJECTILE SHAPE AND CONCRETE PENETRATION DEPTH}

\subsection{Analysis of low speed driving penetration process}

When the driving penetration velocity quasi-static, drill machine is used to load according to drill shape and oval shape penetration projectile. When loading, same axial force and same rotation velocity of driving projectile is given, the input power, axial load, normal torque and penetration depth and other parameters are measured during penetration process according to given time.

Reorganize the measured parameters, and fix the power of driving penetration projectile. When the feed rate is $0.18 \mathrm{~mm} / \mathrm{r}$, the relation between penetration depth of drill shape penetration projectile head and total power at stable phase is obtained as shown in Figure 5.

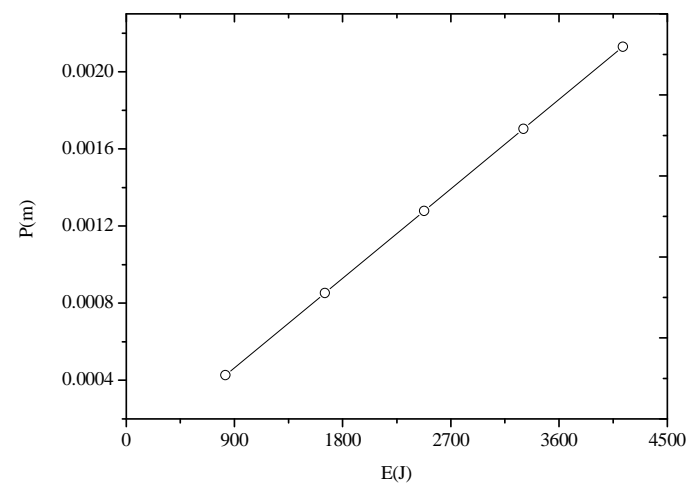

Figure 5. Deductive relation between different input power and penetration depth at low speed The penetration depth of oval shape projectile is 0 ; the penetration results of two shape projectile at low speed show that the drill shape projectile is advanced. It is because that at low speed the drill shape penetration projectile plays the role of rotation effect during penetration process better. Its penetration depth changes slowly, and it only has a better penetration performance at low speed; so we can use the drill shape cutting edge to improve the low speed penetration depth of oval shape projectile.

\subsection{Analysis of high speed driving penetration process}

Propellant is used to load at high speed driving penetration. Firstly, drill shape and oval shape are applied to simulate. Relations between penetration depth of two projectile shapes and penetration velocity is as shown in Figure 6.

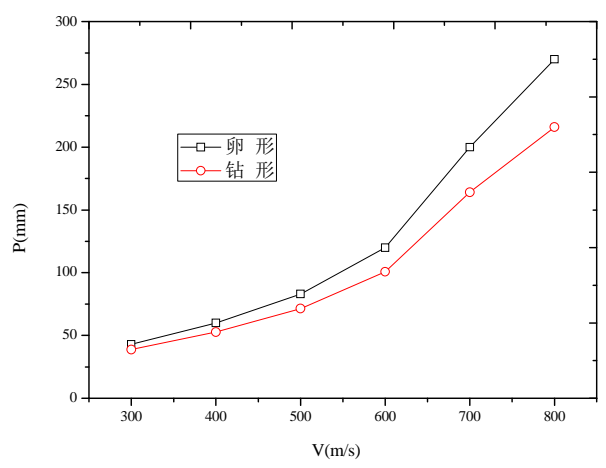

Figure 6. Relation between penetration depth of different shapes and penetration velocity at high speed load

The simulation results show that because the projectile shape coefficient of drill shape penetration projectile is bad, its penetration depth is less than that of oval projectile. It is mainly because that 
the cutting effect reduces during high speed driving penetration, and the axial penetration effect increases. The axial penetration becomes the main influence factor on driving penetration.

\section{CONCLUSIONS}

The research shows that driving penetration is an advanced penetration method, its penetration depth relies on the projectile shape and driving mode.

At low speed, the driving penetration with cutting edge will improve the penetration depth. At high speed, oval shape projectile has more advantages. The oval shape projectile with cutting groove can give full play to the both; when the rotation velocity is $70000 \mathrm{r} / \mathrm{min}$ and the load velocity is about $450 \mathrm{~m} / \mathrm{s}$, the penetration depth of oval shape projectile with cutting groove is maximum.

Under optimal driving loading condition, the influence of groove depth, inclination angle and shape of target face of groove is large. There is a optimal driving projectile shape, in engineering application, the groove depth, inclination angle and area of target face can be appropriately increased to increase driving depth.

\section{REFERENCES}

[1] LI Xiao-jie, JIANG Li,ZHAO Zheng, LIU Da-hui, OU-YANG Xin. Numerical study on penetration of a high-speed-rotating bullet into the moving sheet-metal plate[J]. EXPLOSION AND SHOCK WAVES, 2008, 28(1):57-62.

[2] ZHAO Zi-long, ZHANG Jin-jin, HUANG Xiao-qiong, Revolution effect analysis of a long rod penetrating into soil[J]. JOURNAL OF VIBRATION AND SHOCK, 2010, 29(4):9-11.

[3] PAN Xu-chao, HE Yong, HE Yuan, ZHAO Xiao-ning. Experimental study of penetrating concrete target with a spin-boosted earth penetrating weapon[J]. JOURNAL OF SOLID ROCKET TECHNOLOGY. 2011, 34(2):146-149.

[4] PANG Chun-xu, HE Yong, SHEN Xiao-jun, ZHANG Xian-feng, et al. Experimental Investigation on Penetration of Grooved Projectiles into Concrete Targets. Acta Armamentarii[J]. 2015, 36(1):46-52.

[5] Piekutowski AJ, Forrestal MJ, Poormon KL,et al. Perforation of aluminum plates with ogivenose steel rods at normal and oblique impacts[J]. Int J Impact Eng. 1996,18:877-887.

[6] Johnson G R, Cook W H. Fracture characteristics of three metals subjected to various strains, strains rates, temperatures and pressures[J]. Engineering Fracture Mechanics, 1985, 21(1):31-48. 